\title{
SLANT LIGHTLIKE SUBMANIFOLDS OF AN INDEFINITE SASAKIAN MANIFOLD
}

\author{
JAE WON LeE ${ }^{\mathrm{a}, *}$ AND DAE Ho JiN ${ }^{\mathrm{b}}$
}

\begin{abstract}
In this paper, we introduce the notion of a slant lightlike submanifold of an indefinite Sasakian manifold. We provide a non-trivial example and obtain necessary and sufficient conditions for the existence of a slant lightlike submanifold. Also, we prove some characterization theorems.
\end{abstract}

\section{INTRODUCTION}

The study of lightlike submanifolds of semi-Riemannian geometry appears to fill a gap in the general theory of submanifolds. The main difference between the lightlike submanifolds and non-degenerate submanifolds comes to the fact that the normal vector bundle has non-trivial intersection with the tangent vector bundle. The geometry of lightlike submanifolds of indefinite Kaehler manifolds was presented in a book by Duggal and Bejancu [4]. Chen has introduced the notion of slant immersions by generalizing the concept of holomorphic and totally real immersions [2]. To define the notion of slant submanifolds, one needs to consider the angle between two vector fields. A lightlike submanifold has two (radical and screen) distributions. The radical distribution is totally lightlike and therefore it is impossible to define an angle between two vector fields of radical distribution. On the other hand, the screen distribution is non-degenerate. Using these facts, the notion of slant lightlike submanifold was introduced by Sahin, Gupta and Sharfuddin [8, 9, 11].

The purpose of this paper is to introduce the notion of slant lightlike submanifold of an indefinite Sasakian manifold. In Section 1, we have collected the formulae and information which are useful in our subsequent sections. In Section 2, we introduce the concept of a slant lightlike submanifold of an indefinite Sasakian manifold and

Received by the editors December 25, 2011. Revised April 9, 2012. Accepted April 17, 2012.

2000 Mathematics Subject Classification. 53B25, 53C40, 53C50.

Key words and phrases. slant lightlike submanifold.

${ }^{*}$ Corresponding author. 
provide a non-trivial example. After then, we provide a characterization theorem for the existence of slant lightlike submanifolds and show that co-isotropic $C R$-lightlike submanifolds are slant lightlike submanifolds. Finally, we consider minimal slant ligthlike submanifolds and prove two characterization thoerems.

\section{Preliminaries}

Let $(\bar{M}, \bar{g})$ be a real $(m+n)$-dimensional semi-Riemannian manifold of constant index $q$ such that $m, n \geq 1,1 \leq q \leq m+n-1$ and $(M, g)$ be a submanifold of dimension $m$ of $\bar{M}$. We follow Duggal-Jin [5] for notations and results used in this paper. Throughout this paper we denote by $F(M)$ the algebra of smooth functions on $M$ and by $\Gamma(E)$ the $F(M)$ module of smooth sections of any vector bundle $E$ over $M$. We say that $M$ is a lightlike submanifold of $\bar{M}$ if it admits a degenerate metric $g$ induced from $\bar{g}$. In this case the radical distribution $\operatorname{Rad}(T M)=T M \cap T M^{\perp}$ of $M$ is a vector subbundle of the tangent bundle $T M$ and the normal bundle $T M^{\perp}$, of rank $r$. In general, there exist two complementary non-degenerate distributions $S(T M)$ and $S\left(T M^{\perp}\right)$ of $\operatorname{Rad}(T M)$ in $T M$ and $T M^{\perp}$ respectively, called the screen and co-screen distributions on $M$, such that

$$
T M=\operatorname{Rad}(T M) \oplus_{\text {orth }} S(T M), \quad T M^{\perp}=\operatorname{Rad}(T M) \oplus_{\text {orth }} S\left(T M^{\perp}\right),
$$

where the symbol $\oplus_{\text {orth }}$ denotes the orthogonal direct sum. We denote such a lightlike submanifold by $\left(M, g, S(T M), S\left(T M^{\perp}\right)\right)$. We say that a lightlike submanifold $\left(M, g, S(T M), S\left(T M^{\perp}\right)\right)$ of $\bar{M}$ is

(1) $r$-lightlike if $1 \leq r<\min \{m, n\}$;

(2) co-isotropic if $1 \leq r=n<m$;

(3) isotropic if $1 \leq r=m<n$;

(4) totally lightlike if $1 \leq r=m=n$.

The above three classes $(2) \sim(4)$ are particular cases of the class (1) as follows: $S\left(T M^{\perp}\right)=\{0\}, S(T M)=\{0\}$ and $S(T M)=S\left(T M^{\perp}\right)=\{0\}$ respectively. The geometry of $r$-lightlike submanifolds is more general than that of the other three type submanifolds. For this reason, in this paper we consider only $r$-lightlike submanifolds $M \equiv\left(M, g, S(T M), S\left(T M^{\perp}\right)\right)$.

For the rest of this paper, by a lightlike submanifold we shall mean an r-lightlike submanifold, unless specified.

Let $\operatorname{tr}(T M)$ and $\operatorname{lt}(T M)$ be complementary (but not orthogonal) vector bundles to $T M$ in $T \bar{M}_{\mid M}$ and $T M^{\perp}$ in $S(T M)^{\perp}$ respectively. Then we have 


$$
\begin{aligned}
\operatorname{tr}(T M) & =\operatorname{ltr}(T M) \oplus S\left(T M^{\perp}\right), \\
\left.T \bar{M}\right|_{M} & =T M \oplus \operatorname{tr}(T M) \\
& =(\operatorname{Rad}(T M) \oplus \operatorname{ltr}(T M)) \oplus S(T M) \oplus S\left(T M^{\perp}\right) .
\end{aligned}
$$

We call $\operatorname{tr}(T M)$ and $\operatorname{tr}(T M)$ transversal and lightlike transversal vector bundle of $M$. Consider the following local quasi-orthonormal field of frames of $\bar{M}$ along $M$ :

$$
\left\{\xi_{1}, \ldots, \xi_{r}, N_{1}, \ldots, N_{r}, X_{r+1}, \ldots, X_{m}, W_{r+1}, \ldots, W_{n}\right\}
$$

where $\left\{\xi_{1}, \ldots, \xi_{r}\right\}$ is a lightlike basis of $\Gamma(\operatorname{Rad}(T M)),\left\{N_{1}, \ldots, N_{r}\right\}$ a lightlike basis of $\Gamma(\operatorname{ltr}(T M)),\left\{X_{r+1}, \ldots, X_{m}\right\}$ and $\left\{W_{r+1}, \ldots, W_{n}\right\}$ orthonormal basis of $\Gamma(S(T M) \mid \mathcal{U})$ and $\Gamma\left(S\left(T M^{\perp}\right) \mid \mathcal{U}\right)$ respectively. Then we have

$$
\bar{g}\left(N_{i}, \xi_{j}\right)=\delta_{i j}, \quad \bar{g}\left(N_{i}, N_{j}\right)=0,
$$

where $\left\{\xi_{1}, \ldots, \xi_{r}\right\}$ is a lightlike basis of $\Gamma(\operatorname{Rad}(T M))$.

Let $\bar{\nabla}$ be the Levi-Civita connection on $\bar{M}$. Due to (1.3) we put

$$
\begin{aligned}
& \bar{\nabla}_{X} Y=\nabla_{X} Y+h(X, Y), \forall X, Y \in \Gamma(T M), \\
& \bar{\nabla}_{X} V=-A_{V} X+\nabla_{X}^{\perp} V, \forall X \in \Gamma(T M), V \in \Gamma(\operatorname{tr}(T M)),
\end{aligned}
$$

where $\left\{\nabla_{X} Y, A_{V}\right\}$ and $\left\{h(X, Y), \nabla_{X}^{\perp} V\right\}$ belong to $\Gamma(T M)$ and $\Gamma(\operatorname{tr}(T M))$ respectively. $\nabla$ and $\nabla^{\perp}$ are linear connections on $M$ and $\operatorname{tr}(T M)$ respectively. Besides $\nabla$ is torsion-free linear connection. Also, $h$ is a $\Gamma(\operatorname{tr}(T M))$-value symmetric $F(M)$ bilinear form on $\Gamma(T M)$ and $A_{V}$ is a shape operator on $\Gamma(T M)$. We call $\nabla$ and $\nabla^{\perp}$ the induced connection and the transversal connection on $M$ respectively. Also $h$ is called the second fundamental form of $M$ with respect to $\operatorname{tr}(T M)$. Using (1.2) and (1.3), (1.5) and (1.6) become

$$
\begin{aligned}
& \bar{\nabla}_{X} Y=\nabla_{X} Y+h^{\ell}(X, Y)+h^{s}(X, Y), \\
& \bar{\nabla}_{X} N=-A_{N} X+\nabla_{X}^{\ell} N+D^{s}(X, N), \\
& \bar{\nabla}_{X} W=-A_{W} X+\nabla_{X}^{s} W+D^{\ell}(X, W),
\end{aligned}
$$

for any $X, Y \in \Gamma(T M), N \in \Gamma(\operatorname{ltr}(T M))$ and $W \in \Gamma\left(S\left(T M^{\perp}\right)\right)$. By using $(1.5) \sim$ (1.9) and the fact that $\bar{\nabla}$ is metric, we obtain

$$
\begin{gathered}
\bar{g}\left(h^{s}(X, Y), W\right)+\bar{g}\left(Y, D^{\ell}(X, W)\right)=g\left(A_{W} X, Y\right), \\
\bar{g}\left(h^{\ell}(X, Y), \xi\right)+\bar{g}\left(Y, h^{\ell}(X, \xi)\right)+g\left(Y, \nabla_{X} \xi\right)=0 \\
\bar{g}\left(D^{s}(X, N), W\right)=\bar{g}\left(N, A_{W} X\right),
\end{gathered}
$$




$$
\begin{gathered}
\left.\bar{g}\left(A_{N} X, N^{\prime}\right)+\bar{g}\left(A_{N^{\prime}} X, N\right)\right)=0, \\
\bar{g}\left(A_{N} X, P Y\right)=\bar{g}\left(N, \bar{\nabla}_{X} P Y\right),
\end{gathered}
$$

for any $\xi \in \Gamma(\operatorname{Rad}(T M)), W \in \Gamma\left(S\left(T M^{\perp}\right)\right.$ and $N, N^{\prime} \in \Gamma(\operatorname{ltr}(T M))$.

The induced connection $\nabla$ on $T M$ is not metric and satisfies

$$
\left(\nabla_{X} g\right)(Y, Z)=\sum_{i=1}^{r}\left\{h_{i}^{\ell}(X, Y) \eta_{i}(Z)+h_{i}^{\ell}(X, Z) \eta_{i}(Y)\right\},
$$

for all $X, Y \in \Gamma(T M)$, where $\eta_{i}$ s are the $r$ differential 1-forms such that

$$
\eta_{i}(X)=\bar{g}\left(X, N_{i}\right), \forall X \in \Gamma(T M) .
$$

But the connection $\nabla^{*}$ on $S(T M)$ is metric. Denote by $P$ the projection morphism of $T M$ on $S(T M)$ with respect to (1.1). According to (1.1) we set

$$
\begin{aligned}
& \nabla_{X} P Y=\nabla_{X}^{*} P Y+h^{*}(X, P Y), \\
& \nabla_{X} \xi=-A_{\xi}^{*} X+\nabla_{X}^{* t} \xi,
\end{aligned}
$$

for any $X, Y \in \Gamma(T M)$ and $\xi \in \Gamma(\operatorname{Rad}(T M))$, where the sets $\left\{\nabla_{X}^{*} P Y, A_{\xi}^{*} X\right\}$ and $\left\{h^{*}(X, P Y), \nabla_{X}^{* t} \xi\right\}$ belong to $\Gamma(S(T M))$ and $\Gamma(\operatorname{Rad}(T M))$ respectively. It follows that $\nabla^{*}$ and $\nabla^{* t}$ are linear connections on complementary distributions $S(T M)$ and $\operatorname{Rad}(T M)$ respectively. On the other hand, $h^{*}$ is $\Gamma(\operatorname{Rad}(T M))$-valued $F(M)$-bilinear form on $\Gamma(T M) \times \Gamma(S(T M))$ and $A_{\xi}^{*}$ is a linear operator on $\Gamma(T M)$. Call $h^{*}$ the second fundamental form of $S(T M)$ and $A^{*}$ the shape operator of $S(T M)$ with respect to $\xi$. Also, call $\nabla^{*}$ and $\nabla^{* t}$ the induced connections on $S(T M)$ and $\operatorname{Rad}(T M)$ respectively. It is important to note that both $\nabla^{*}$ and $\nabla^{* t}$ are metric connections. The second fundamental form and the shape operator of a non-degenerate submanifold of a semi-Riemannian manifold are related by means of the metric tensor field (see Chen[1]). Contrary to this, in the lightlike case there are interrelations between geometric objects induced by $\operatorname{tr}(T M)$ and $S(T M)$. More precisely, by using (1.7), (1.17) and (1.18) we obtain

$$
\begin{aligned}
& \bar{g}\left(h^{\ell}(X, P Y), \xi\right)=\bar{g}\left(A_{\xi}^{*} X, P Y\right), \\
& \bar{g}\left(h^{*}(X, P Y), N\right)=\bar{g}\left(A_{N} X, P Y\right),
\end{aligned}
$$

for any $X, Y \in \Gamma(T M), \xi \in \Gamma(\operatorname{Rad}(T M))$ and $N \in \Gamma(\operatorname{ltr}(T M))$. As $h^{\ell}$ is symmetric, from (1.19) it follows that the shape operator of $S(T M)$ is a self-adjoint operator on $S(T M)$, i.e., we have

$$
g\left(A_{\xi}^{*} P X, P Y\right)=g\left(P X, A_{\xi}^{*} P Y\right), \forall X, Y \in \Gamma(T M) .
$$


Replace $Y$ by $\xi$ in (1.11) we deduce

$$
\bar{g}\left(h^{\ell}(X, \xi), \xi\right)=0, \forall X \in \Gamma(T M) .
$$

Then replace $X$ by $\xi$ in (1.19) and by using (1.21), we obtain

$$
A_{\xi}^{*} \xi=0 .
$$

By using the linear connections introduced by (1.9) (1.11), we use the following covariant derivatives:

$$
\begin{aligned}
& \left(\nabla_{X} h^{\ell}\right)(Y, Z)=\nabla_{X}^{\ell}\left(h^{\ell}(Y, Z)\right)-h^{\ell}\left(\nabla_{X} Y, Z\right)-h^{\ell}\left(Y, \nabla_{X} Z\right), \\
& \left(\nabla_{X} h^{s}\right)(Y, Z)=\nabla_{X}^{s}\left(h^{s}(Y, Z)\right)-h^{s}\left(\nabla_{X} Y, Z\right)-h^{s}\left(Y, \nabla_{X} Z\right),
\end{aligned}
$$

for $X, Y, Z \in \Gamma(T M), \xi \in \Gamma(\operatorname{Rad}(T M))), N \in \Gamma(l \operatorname{tr}(T M))$ and $W \in \Gamma\left(S\left(T M^{\perp}\right)\right)$.

An odd dimensional semi-Riemannian manifold $(\bar{M}, \bar{g})$ is called a contact metric manifold $[6,7]$ if there exists a $(1,1)$-type tensor field $\varphi$, a vector field $V$, called the characteristic vector field, and its 1 -form $\theta$ satisfying

$$
\begin{aligned}
& \varphi^{2} X=-X+\eta(X) V, \varphi V=0, \eta \circ \varphi=0, \eta(V)=1, \\
& \bar{g}(V, V)=\epsilon, \quad \bar{g}(\varphi X, \varphi Y)=\bar{g}(X, Y)-\epsilon \eta(X) \eta(Y), \\
& \eta(X)=\epsilon \bar{g}(V, X), \quad d \eta(X, Y)=\bar{g}(\varphi X, Y), \epsilon= \pm 1,
\end{aligned}
$$

for any $X, Y \in \Gamma(T \bar{M})$. Then $(\varphi, \eta, V, \bar{g})$ is called a contact metric structure on $\bar{M}$. We say that $\bar{M}$ has a normal contact structure if $N_{\varphi}+d \theta \otimes V=0$, where $N_{\varphi}$ is the Nijenhuis tensor field of $\varphi[6,7]$. A normal contact metric manifold is called a Sasakian manifold [12] for which we have

$$
\begin{gathered}
\bar{\nabla}_{X} V=\varphi X \\
\left(\bar{\nabla}_{X} \varphi\right) Y=\epsilon \eta(Y) X-\bar{g}(X, Y) V .
\end{gathered}
$$

The next ingredient we consider is a semi-Riemannian metric $\bar{g}$ of index $\mu(>0)$ on the Sasakian manifold $\bar{M}=(\bar{M}, \varphi, V, \eta, \bar{g})$. Then we say that $\bar{M}$ is an indefinite Sasakian manifold. In an indefinite Sasakian manifold $\bar{M}$, the characteristic vector field $V$ is a spacelike vector field on $\bar{M}[10]$.

A general notion of a minimal lightlike submanifold in a semi-Riemannian manifold, as introduced by Bejancu and Duggal [4], is as follows:

Definition 1.1. A lightlike submanifold $(\bar{M}, \bar{g}, S(T M))$ isometrically immersed in a semi-Riemannian manifold $(\bar{M}, \bar{g})$ is said to be minimal if

(1) $h^{s}=0$ on $\operatorname{Rad}(T M)$;

(2) traceh $=0$, when trace is written with respect to $g$ restricted to $S(T M)$. 
Similar to the definition for a contact $C R$-lightlike submanifold of indefinite Sasakian manifold [7], we state the following:

Definition 1.2. Let $\left(M, g, S(T M), S\left(T M^{\perp}\right)\right.$ be a lightlike submanifold and immersed in an indefinite Sasakian manifold $(\bar{M}, \bar{g})$. We say hat $M$ is a contact $C R$ lightlike submanifold of $\bar{M}$ if the following conditions are satisfied:

(1) $\varphi \operatorname{Rad}(T M)$ is a distribution on $M$ such that $\operatorname{Rad}(T M) \cap \varphi(\operatorname{Rad}(T M))=\{0\}$;

(2) There exist vector bundles $\mathcal{D}_{0}$ and $\mathcal{D}^{\prime}$ over $M$ such that

$$
\left\{\begin{array}{l}
S(T M)=\left\{\varphi(\operatorname{Rad}(T M)) \oplus \mathcal{D}^{\prime}\right\} \perp \mathcal{D}_{0} \perp\{\zeta\} \\
\varphi \mathcal{D}_{0}=\mathcal{D}_{0} \\
\varphi \mathcal{D}^{\prime}=L_{1} \perp \operatorname{ltr}(T M),
\end{array}\right.
$$

where $\perp$ is the orthogonal direct sum, $\mathcal{D}_{0}$ is nondegenerate and $L_{1}$ is a vector subbundle of $S\left(T M^{\perp}\right)$. A contact $C R$-lightlike submanifold is proper if $\mathcal{D}_{0} \neq\{0\}$ and $L_{1} \neq\{0\}$.

Example 1.3 ([7]). Let $M$ be a lightlike hypersurface of $\bar{M}$. Then $M$ is a contact $C R$-lightlike hypersurface.

\section{Slant Lightlike Submanifolds}

Lemma 2.1. Let $M$ be an r-lightlike submanifoldof an indefinite Sasakian manifold $\bar{M}$ of index $2 q$. Suppose that $\varphi(\operatorname{Rad}(T M))$ is a distribution on $M$ such that $\operatorname{Rad}(T M) \cap \varphi(\operatorname{Rad}(T M))=\{0\}$. Then $\varphi(\operatorname{Rad}(T M))$ is a subbundle of the screen distribution $S(T M)$ and $\varphi(\operatorname{ltr}(T M)) \cap \varphi(\operatorname{Rad}(T M))=\{0\}$.

Proof. By hypothesis, since $\varphi(\operatorname{Rad}(T M))$ is a distribution on $M$ such that

$$
\operatorname{Rad}(T M) \cap \varphi(\operatorname{Rad}(T M))=\{0\},
$$

we have $\varphi(\operatorname{Rad}(T M)) \in S(T M)$. Choose $N \in \operatorname{ltr}(T M), \xi \in \operatorname{Rad}(T M), X \in$ $S(T M)$, and $W \in S\left(T M^{\perp}\right)$ such that $\bar{g}(N, \xi)=\bar{g}(X, X)=\bar{g}(W, W)=1$, we can write that

$$
\varphi N=k_{1} N+k_{2} \xi+k_{3} X+k_{4} W
$$

where $k_{1}, k_{2}, k_{3}$, and $k_{4}$ are smooth functions on $M$. Taking the scalar product of (2.1) with $N$ and $\xi$, we get $k_{2}=0$ and $k_{1}=0$, respectively. Thus we have

$$
\varphi N=k_{3} X+k_{4} W
$$


Let us suppose that $\varphi N$ belongs to $S\left(T M^{\perp}\right)$. Then we have $1=\bar{g}(\xi, N)=$ $\bar{g}(\varphi \xi, \varphi N)=0$ due to $\left.\varphi N \in \Gamma\left(S\left(T M^{\perp}\right)\right)\right)$ and $\varphi \xi \in \Gamma(S(T M))$, which is a contradiction. Therefore, from (2.2) we conclude $\varphi N$ belongs to $S(T M)$ and $\varphi(\operatorname{ltr}(T M))$ is a distribution on $\mathrm{M}$.

Moreover, $\varphi N$ dose not belong to $\varphi(\operatorname{Rad}(T M))$. Indeed if $\varphi N \in \Gamma(\operatorname{Rad}(T M))$, we would have $\varphi^{2} N=-N+\theta(N) V=-N \in \Gamma(\operatorname{Rad}(T M))$, but this is impossible. Thus, we conclude $\varphi(\operatorname{ltr}(T M)) \subset S(T M)$ and $\varphi(\operatorname{ltr}(T M)) \cap \varphi(\operatorname{Rad}(T M))=\{0\}$.

Lemma 2.2. Let $M$ be q-lightlike submanifold of an indefinite Sasakian manifold $\bar{M}$ of index $2 q$ with the characteristic field tangent to $M$. Suppose that $\varphi(\operatorname{Rad}(\operatorname{TM}))$ is a distribution on $M$ such that $\operatorname{Rad}(T M) \cap \varphi(\operatorname{Rad}(T M))=\{0\}$. Then any complementary distribution to $\varphi(\operatorname{ltr}(T M)) \oplus \varphi(\operatorname{Rad}(T M))$ in the screen distribution $S(T M)$ is Reimannian.

Proof. Let $D^{\prime}$ be the complemantary distribution to $\varphi(\operatorname{ltr}(T M)) \oplus \varphi(\operatorname{Rad}(T M)) \subset$ $S(T M)$ and let $\operatorname{dim}(\bar{M})=m+n$ and $\operatorname{dim}(M)=m$. We can choose a local quasi orthornomal frame on $\bar{M}$ along $M$ as follows:

$$
\begin{aligned}
&\left\{\xi_{i}, N_{i}, \varphi \xi_{i}, \varphi N_{i}, X_{\alpha}, V, W_{\beta}\right\}, i \in\{1, \ldots, q\}, \\
& \alpha \in\{3 q+1, \ldots, m-1\}, \quad \beta \in\{q+1, \ldots, n\},
\end{aligned}
$$

where $\left\{\xi_{i}\right\}$ and $\left\{N_{i}\right\}$ are lightlike basis of $\operatorname{Rad}(T M)$ and $\operatorname{ltr}(T M)$, respectively, and $\left\{\varphi \xi_{i}, \varphi N_{i}, X_{\alpha}, V\right\}$ is an orhonormal basis of $S(T M)$ and $\left\{W_{\beta}\right\}$ is an orthonormal basis of $S\left(T M^{\perp}\right)$. Now, we construct an orthonormal basis $\left\{U_{1}, \ldots, U_{2 q}, V_{1}, \ldots, V_{2 q}\right\}$ as follows:

$$
\begin{array}{cc}
U_{1}=\frac{1}{\sqrt{2}}\left\{\xi_{1}+N_{1}\right\} & U_{2}=\frac{1}{\sqrt{2}}\left\{\xi_{2}-N_{2}\right\}, \\
U_{3}=\frac{1}{\sqrt{2}}\left\{\xi_{3}+N_{3}\right\} & U_{4}=\frac{1}{\sqrt{2}}\left\{\xi_{4}-N_{4}\right\}, \\
\ldots & \ldots \\
\ldots & \ldots \\
U_{2 q-1}=\frac{1}{\sqrt{2}}\left\{\xi_{q}+N_{q}\right\} & U_{2 q}=\frac{1}{\sqrt{2}}\left\{\xi_{q}-N_{q}\right\}, \\
V_{1}=\frac{1}{\sqrt{2}}\left\{\varphi \xi_{1}+\varphi N_{1}\right\} & V_{2}=\frac{1}{\sqrt{2}}\left\{\varphi \xi_{2}-\varphi N_{2}\right\}, \\
\ldots & \ldots
\end{array}
$$




$$
V_{2 q-1} 1=\frac{1}{\sqrt{2}}\left\{\varphi \xi_{q}+\varphi N_{q}\right\} \quad V_{2 q}=\frac{1}{\sqrt{2}}\left\{\varphi \xi_{q}-\varphi N_{q}\right\} .
$$

Hence, $\left\{\xi_{i}, N_{i}, \varphi \xi_{i}, \varphi N_{i}\right\}$ gives a non-degenerate space of constant index $2 q$ which implies that $\operatorname{Rad}(T M) \oplus \operatorname{ltr}(T M) \oplus \varphi(\operatorname{Rad}(T M)) \oplus \varphi(\operatorname{ltr}(T M))$ is nod-degenerate and of constant index $2 q$ on $\bar{M}$. As

$$
\begin{aligned}
\operatorname{index}(T \bar{M})= & \operatorname{index}(\operatorname{Rad}(T M) \oplus \operatorname{ltr}(T M)) \\
& +i \operatorname{index}(\varphi(\operatorname{Rad}(T M)) \oplus \varphi(\operatorname{ltr}(T M))) \\
& +i n d e x\left(D^{\prime} \oplus S\left(T M^{\perp}\right)\right),
\end{aligned}
$$

we have

$$
2 q=2 q+\operatorname{index}\left(D^{\prime} \oplus S\left(T M^{\perp}\right)\right),
$$

which implies that index $\left(D^{\prime} \perp S\left(T M^{\perp}\right)\right)=0$. Hence $D^{\prime}$ is Riemannian.

To define slant lightlike submanifolds of indefinite Sasakian manifolds, one needs to consider an angle between two vector fields. We shown from Section 1 that a lightlike submanifold has two(radical and screen) distributions. The radical distribution is totally lightlike and therefore it is not impossilbe to define angle between two vector fields of radical distribution. On the other hand, the screen distribution is non-degenerate. Thus one way to define slant lightlike submanifolds is to choose a Riemannian screen distribution on lightlike submanifolds, for which we use Lemma 2.2 .

Definition 2.3. Let $M$ be a $q$-lightlike submaifold of an indefinite Sasakian manifold $\bar{M}$ of index $2 q$ with $V$ tangent to $M$. Then we say that $M$ is a slant lightlike submanifold of $\bar{M}$ if the following conditions are satisfied:

(i) $\varphi \operatorname{Rad}(T M)$ is a distribution on $M$ such that $\operatorname{Rad}(T M) \cap \varphi(\operatorname{Rad}(T M))=$ $\{0\}$.

(ii) For all $x \in \mathcal{U} \subset M$ and for each non-zero vector field $X$ tangent to $\bar{D}=D \perp$ $\{V\}$, if $X$ and $V$ are linearly independent, then the angle $\theta(X)$ between $\varphi X$ and the vector space $\bar{D}_{x}$ is constant, where $D$ is complementary distribution to $\varphi(\operatorname{ltr}(T M)) \oplus \varphi(\operatorname{Rad}(T M))$ in screen distribution $S(T M)$.

The constant angle $\theta(X)$ is called the slant angle of $\bar{D}$. A slant lightlike submanifold $M$ is said to be proper if $D \neq\{0\}$ and $\theta \neq 0, \frac{\phi}{2}$.

If $M$ is totally lightlike submanifold of $\bar{M}$, then we have $T M=\operatorname{Rad}(T M)$, and hence $D=\{0\}$. Therefore we have the following: 
Proposition 2.4. There exist no proper slant totally lightlike or isotropic submanifold $M$ in indefinite Sasakian manfiold $\bar{M}$ with the characteristic vector field $V$ tangent to $M$.

From now on, $\left(\mathbb{R}_{q}^{2 m+1}, \varphi_{0}, V, \eta, \bar{g}\right)$ will denote the manifold $\mathbb{R}_{q}^{2 m+1}$ with its usual Sasakian structure given by

$$
\begin{aligned}
& \eta= \frac{1}{2}\left(d z-\sum_{i=1}^{m} y^{i} d x^{i}\right), \quad V=2 \partial z \\
& \bar{g}= \eta \otimes \eta \\
&+\frac{1}{4}\left\{-\sum_{i=1}^{q}\left(d x^{i} \otimes d x^{i}+d y^{i} \otimes d y^{i}\right)+\sum_{i=q+1}^{m}\left(d x^{i} \otimes d x^{i}+d y^{i} \otimes d y^{i}\right)\right\}, \\
& \varphi_{0}\left(\sum_{i=1}^{m}\left(X_{i} \partial x^{i}+Y_{i} \partial y^{i}\right)+Z \partial z\right)=\sum_{i=1}^{m}\left(Y_{i} \partial x^{i}-X_{i} \partial y^{i}\right)+\sum_{i=1}^{m} Y_{i} y^{i} \partial z,
\end{aligned}
$$

where $\left(x^{i}, y^{i}, z\right)$ are the Cartesian coordinates.

Example 1. Let $\bar{M}=\left(\mathbb{R}_{2}^{9}, \bar{g}\right)$ be a semi-Euclidean space, where $\bar{g}$ is of signature $(-,+,+,-,+,+,+,+)$ with respect to canonical basis

$$
\left\{\partial x_{1}, \partial x_{2}, \partial x_{3}, \partial x_{4}, \partial y_{1}, \partial y_{2}, \partial y_{3}, \partial y_{4}, \partial z\right\}
$$

Suppose $M$ is a submanifold of $\mathbb{R}_{2}^{9}$ defined by

$$
x^{1}=y^{4}, \quad x^{2}=\sqrt{1-\left(y^{2}\right)^{2}}, \quad y^{2} \neq \pm 1
$$

It is easy to see that a local frame of $T M$ is given by

$$
\begin{aligned}
& Z_{1}=2\left(\partial x_{1}+\partial y_{4}+y^{1} \partial z\right), \quad Z_{2}=2\left(\partial x_{4}-\partial y_{1}+y^{4} \partial z\right) \\
& Z_{3}=\partial x_{3}+\partial y_{2}+y^{3} \partial z, \quad Z_{4}=\partial y_{1}+2 \partial y_{3} \\
& Z_{5}=-\frac{y^{2}}{x^{2}} \partial x_{2}+\partial y_{2}-\frac{\left(y^{2}\right)^{2}}{x_{2}} \partial z, Z_{6}=\partial x_{4}+\partial y_{1}, \partial Z_{7}=V=2 \partial z
\end{aligned}
$$

Hence, we show that $\operatorname{Rad}(T M)=\operatorname{span}\left\{Z_{1}\right\}, \varphi_{0}(\operatorname{Rad}(T M))=\operatorname{span}\left\{Z_{2}\right\}$, and $\operatorname{Rad}(T M) \cap \varphi_{0}(\operatorname{Rad}(T M))=\{0\}$, hence $(i)$ holds. Next, $\overline{\mathcal{D}}=\mathcal{D} \perp\{V\}=$ $\left\{Z_{3}, Z_{4}\right\} \perp\{V\}$ is Riemannian, where $\perp$ is the orthogonal direct sum. By direct calculations, we get

$$
S\left(T M^{\perp}\right)=\operatorname{span}\left\{W=\partial x_{2}+\frac{y^{2}}{x^{2}} \partial y_{2}+y^{2} \partial z\right\} \text { such that } \varphi_{0}(W)=-Z_{5},
$$

and $\operatorname{lt}(T M)=\operatorname{span}\left\{N=-\partial x_{1}+\partial y_{4}-y \partial z\right\}$ such that $\varphi_{0}(N)=Z_{6}$. Next, we have $\bar{D}=D \perp V=\left\{Z_{3}, Z_{4}, Z_{5}\right\} \perp\{V\}$ is Riemannian, where $\perp$ is the orthogonal direct sum. Then $M$ is proper slant lightlike. 
Proposition 2.5. Slant lightlike submanifolds $M$ of an indefinite Sasakian manifold $\bar{M}$ with the characteristic vector field $V$ tangent to $M$ do not include invariant and screen real lightlike submanifolds.

Proposition 2.6. Let $M$ be a q-lightlike submanifold of an indefinite Sasakian manifold $\bar{M}$ of index $2 q$. Then any coisotropic $C R$-lightlike submanifold is a slant lightlike submanifold with $\theta=0$. In particular, a lightlike real hypersurface of an indefinite Sasakian manifold $\bar{M}$ of index 2 is a slant lightlike submanifold with $\theta=0$. Moreover, any CR-lightlike submanifold of $\bar{M}$ with $D_{0}=\{0\}$ is a slant lightlike submanifold with $\theta=\frac{\pi}{2}$.

Proof. Let $M$ be a $q$-lightlike $C R$-lightlike submanifold of an indefinite Sasakian manifold $\bar{M}$. Then $\varphi(\operatorname{Rad}(T M))$ is a distribution on $M$ such that $\operatorname{Rad}(T M) \cap$ $\varphi(\operatorname{Rad}(T M))=\{0\}$. If $M$ is coisotropic, then $S\left(T M^{\perp}\right)=\{0\}$. Then the complementary distribution to $\varphi(\operatorname{ltr}(T M)) \cap \varphi(\operatorname{Rad}(T M))$ is the screen distribution $S(T M)$ is $\overline{\mathcal{D}}=\mathcal{D}_{0} \perp\{V\}$ where $\mathcal{D}_{0}$ is Riemannian by Lemma 2.2. Since $\mathcal{D}_{0}$ is invariant with respect to $\varphi$, it follows that $V=0$. The second assertion is obvious as a lightlike real hypersurface on $\bar{M}$ is coisotropic. Now, if $M$ is $C R$-lightlike submanifold with $\mathcal{D}_{0}=\{0\}$, then the complementary distribution to $\varphi(\operatorname{ltr}(T M)) \cap \varphi(\operatorname{Rad}(T M))$ in the screen distribution $S(T M)$ is $\overline{\mathcal{D}}=\mathcal{D}^{\prime} \perp\{V\}$. Since $\mathcal{D}^{\prime}$ is anti-invariant with respect to $\varphi$, it follows that $\theta=\frac{\pi}{2}$, which completes the proof.

We know that for any $X \in T M$ and $W \in \operatorname{tr}(T M)$,

$$
\varphi X=T X+F X, \quad \varphi W=B W+C W,
$$

$T X$ and $F X$ are the tangential and transversal components of $\phi X$, respectively and $B W$ and $C W$ are tangential and transversal components of $\phi W$, respectively. Morveover, for a slant lightlike submanifold, we denote by $P_{1}, P_{2}, Q_{1}$, and $Q_{2}$ and $\bar{Q}_{2}$ the projections on the distributions $\operatorname{Rad}(T M), \varphi(\operatorname{Rad}(T M)), \varphi(\operatorname{ltr}(T M)), \mathcal{D}$ and $\overline{\mathcal{D}}=\mathcal{D} \perp\{V\}$, respectively. Then for any $X \in T M$, we can write

$$
X=P_{1} X+P_{2} X+Q_{1} X+\bar{Q}_{2} X,
$$

where $\bar{Q}_{2} X=Q_{2} X+\theta(X) V$. Using (2.5) in the above equation, we obtain

$$
\varphi X=\varphi P_{1} X+\varphi P_{2} X+T Q_{2} X+F Q_{1} X+F Q_{2} X, \quad \forall X \in T M .
$$

Then the tangential components are

$$
T X=T P_{1} X+T P_{2} X+T Q_{2} X .
$$


We now prove two characterization theorems for slant lightlike submanifolds.

Theorem 2.7. Let $M$ be a q-lightlike submanifold of an indefinite Sasakian manifold $\bar{M}$ of index $2 q$ with the characteristic vector field tangent to $M$. Then $M$ is slant lightlike submanifold if and only if the following conditions are satisfied:

(a) $\varphi(l \operatorname{tr}(T M))$ is a distribution on $M$.

(b) There exist a constant $\lambda \in[-1,0]$ such that

$$
T^{2} \bar{Q}_{2} X=\lambda\left(\bar{Q}_{2} X-\theta\left(\bar{Q}_{2} X\right) V\right),
$$

for all $X \in \Gamma(T M)$ linearly independent of the characteristic vector field $V$. Moreover, in such a case, $\lambda=-\cos ^{2} \theta$ when $\theta$ is the slant angle of $M$.

Proof. Let $M$ be a $q$-lightlike submanifold of an indefinite Saskian manifold $\bar{M}$ of index $2 q$. If $M$ is a slant lightlike submanifold of $\bar{M}$, then $\varphi(\operatorname{Rad}(T M))$ is a distribution on $S(T M)$, and hence from Lemma 2.2, it follows that $\varphi(\operatorname{lt}(T M))$ is also a distribution on $M$ and $\varphi(\operatorname{ltr}(T M)) \subset S(T M)$. Thus $(a)$ is complete. For $X \in \Gamma(T M), Q_{2} X \in \overline{\mathcal{D}}-\{V\}$, we have

$$
\begin{aligned}
\cos \theta\left(Q_{2} X\right) & =\frac{\bar{g}\left(\varphi Q_{2} X, T Q_{2} X\right)}{\left|\varphi Q_{2} X\right|\left|T Q_{2} X\right|} \\
& =-\frac{\bar{g}\left(Q_{2} X, \varphi T Q_{2} X\right)}{\left|\varphi Q_{2} X\right|\left|T Q_{2} X\right|} \\
& =-\frac{\bar{g}\left(Q_{2} X, T^{2} Q_{2} X\right)}{\left|Q_{2} X\right|\left|T Q_{2} X\right|}
\end{aligned}
$$

On the other hand, $\cos \theta(X)=\frac{|T X|}{|\varphi X|}$, and so, by using 2.9 , we obtain

$$
\cos ^{2} \theta\left(Q_{2} X\right)=-\frac{\bar{g}\left(Q_{2} X, T^{2} Q_{2} X\right)}{\left|Q_{2} X\right|^{2}} .
$$

Since $\theta\left(Q_{2} X\right)$ is constant on $\overline{\mathcal{D}}$, we conclude that

$$
T^{2} \bar{Q}_{2} X=\lambda Q_{2} X=\lambda\left(\bar{Q}_{2} X-\theta\left(\bar{Q}_{2} X\right) V\right), \quad \lambda \in(-1,0) .
$$

Moreover, in this case, $\lambda=-\cos ^{2} \theta$. It is clear that the above equation is valid for $\theta=0$ and $\theta=\frac{\pi}{2}$. Hence for $\bar{Q}_{2} X \in \overline{\mathcal{D}}$, the proof is complete. Conversely, suppose that $(a)$ and $(b)$ hold. Then $(a)$ implies that $\varphi(\operatorname{Rad}(T M))$ is a distribution on $M$. From Lemma 2.2, it follows that the complementary distribution to $\varphi(\operatorname{lt}(T M)) \oplus$ $\varphi(\operatorname{Rad}(T M))$ is a Riemannian distribution. The rest of the proof is clear.

Corollary 2.8. Let $M$ be a slant submanifold of an indefinite Sasakian manifold $\bar{M}$ of index $2 q$ with the characteristic vector field tangent to $M$. Then, for any 
$X, Y \in \Gamma(T M)$, we have

$$
\begin{aligned}
& g\left(T \bar{Q}_{2} X, T \bar{Q}_{2} Y\right)=\cos ^{2} \theta\left\{g\left(\bar{Q}_{2} X, \bar{Q}_{2} Y\right)-\theta\left(\bar{Q}_{2} X\right) \theta\left(\bar{Q}_{2} Y\right)\right\}, \\
& g\left(F \bar{Q}_{2} X, F \bar{Q}_{2} Y\right)=\sin ^{2} \theta\left\{g\left(\bar{Q}_{2} X, \bar{Q}_{2} Y\right)-\theta\left(\bar{Q}_{2} X\right) \theta\left(\bar{Q}_{2} Y\right)\right\} .
\end{aligned}
$$

Proof. From $g(T X, Y)=-g(X, T Y)$ for all $X \in \Gamma(T M)$ and Theorem 2.7, a direct expension gives (2.10). To prove (2.11), it is enough to take into account (1.19) and $(2.5)$.

Theorem 2.9. Let $M$ be a q-lightlike submanifold of an indefinite Sasakian manifold $\bar{M}$ of index $2 q$ with the characteristic vector field tangent to $M$. Then $M$ is slant lightlike submanifold if and only if the following conditions are satisfied:

(a) $\varphi(\operatorname{lt}(T M))$ is a distribution on $M$.

(b) There exist a constant $\mu \in[-1,0]$ such that

$$
B F \bar{Q}_{2} X=\mu\left(\bar{Q}_{2} X-\theta\left(\bar{Q}_{2} X\right) V\right), \quad \forall X \in \Gamma(T M) .
$$

Moreover, in such a case, $\mu=-\sin ^{2} \theta$ when $\theta$ is the slant angle of $M$.

Proof. It is clear to see that $\varphi(\operatorname{Rad}(T M)) \cap \varphi(l \operatorname{tr}(T M))=\{0\}$ and $\varphi(\operatorname{Rad}(T M))$ is subbundle of $S(T M)$. Moreover, the complementary distribution to $\varphi(\operatorname{lt}(T M)) \oplus$ $\varphi(\operatorname{Rad}(T M))$ in $S(T M))$ is Riemannian. Furthermore, from the proof of Lemma 2.2, $S\left(T M^{\perp}\right)$ is also Riemannian. Thus $(i)$ in the Definition 2.3 of slant lightlike submanifold is satisfied. On the other hand, from (2.5) and (2.7), we obtain

$$
-X=-P_{1} X-P_{2} X+T^{2} Q_{2} X+F T Q_{2} X+J F Q_{1} X+B F Q_{2} X+C F Q_{2} X .
$$

Since $\varphi F Q_{1} X=-Q_{1} X \in \Gamma(S(T M))$, takining the tangential parts, we have

$$
-X+\theta(X) V=-P_{1} X-P_{2} X+T^{2} Q_{2} X-Q_{1} X+B F Q_{2} X .
$$

From (2.6), we obtain

$$
-Q_{2} X=-T^{2} Q_{2} X+B F Q_{2} X .
$$

Now, if $M$ is slant lightlike, then from Theorem 2.7, we have $T^{2} Q_{2} X=-\cos ^{2} \theta Q_{2} X$, and hence we get $B F Q_{2} X=-\sin ^{2} \theta Q_{2} X$. Since $F V=0$ and $\bar{Q}_{2} X=Q_{2} X+\theta(X) V$, we have $B F \bar{Q}_{2} X=-\sin ^{2} \theta\left\{\bar{Q}_{2} X-\theta\left(\bar{Q}_{2} X\right) V\right\}$.

Conversely, suppose that $B F Q_{2} X=\mu Q_{2} X$. Then, from (2.12), we obtain

$$
T^{2} Q_{2} X=-(1+\mu) Q_{2} X \text {. }
$$

Thus, the proof follows from Theorem 2.7. 


\section{Minimal Slant Lightlike Submanifolds}

Now we study minimal slant lightlike submanifolds of indefinite Sasakian manifolds. In what follows, we prove two characterization results for minimal slant lightlike submanifolds. First we give the following lemma.

Lemma 3.1. Let $M$ be a proper slant lightlike submanifold of an indefinite Sasakian manifold $\bar{M}$ such that $\operatorname{dim}(\mathcal{D})=\operatorname{dim}\left(S\left(T M^{\perp}\right)\right)$. If $\left\{e_{1}, \ldots, e_{m}\right\}$ is a local orthonormal basis of $\Gamma(\mathcal{D})$, then $\left\{\csc \theta F e_{1}, \ldots, \csc \theta F e_{m}\right\}$ is an orthonormal basis of $S\left(T M^{\perp}\right)$.

Proof. Since $\left\{e_{1}, \ldots, e_{m}\right\}$ is a local orthonormal basis for $\mathcal{D}$ and $\mathcal{D}$ is Riemannian, from Corollary 2.8, we find

$$
\bar{g}\left(\csc \theta F e_{i}, \csc \theta F e_{j}\right)=\delta_{i j},
$$

where $i, j=1,2, \ldots, m$, which proves the assertion.

Theorem 3.2. Let $M$ be a proper slant lightlike submanifold of an indefinite Sasakian manifold $\bar{M}$ with the characteristic vector field tangent to $M$. Then $M$ is minimal if and only if

$$
\left.\operatorname{trace} A_{W_{j}}\right|_{S(T M)}=0,\left.\quad \operatorname{trace} A_{\xi_{k}}^{*}\right|_{S(T M)}=0, \quad \text { and } \quad \bar{g}\left(D^{l}(X, W), Y\right)=0,
$$

for $X, Y \in \Gamma(\operatorname{Rad}(T M)), W \in \Gamma\left(S\left(T M^{\perp}\right)\right)$, where $\left\{\xi_{k}\right\}_{k=1}^{r}$ is a basis of $\operatorname{Rad}(T M)$ and $\left\{W_{j}\right\}_{j=1}^{r}$ is a basis of $S\left(T M^{\perp}\right)$.

Proof. From (1.25), we have $\bar{\nabla}_{V} V=0$ and thus from (1.3) we get $h^{l}(V, V)=$ $h^{s}(V, V)=0$. Now, take an orthonormal frame basis of $S\left(T M^{\perp}\right)$ of $\mathcal{D}$.

From (1.16), we know $h_{i}^{l}=0$ on $\operatorname{Rad}(T M)$ for all $i$. Thus, $M$ is minimal if and only if

$$
\sum_{k=1}^{r} h\left(\varphi \xi_{k}, \varphi \xi_{k}\right)+\sum_{k=1}^{r} h\left(\varphi N_{k}, \varphi N_{k}\right)+\sum_{i=1}^{m} h\left(e_{i}, e_{i}\right)=0 .
$$

Using (1.10) and (1.19), we obtain

$$
\begin{aligned}
& \sum_{k=1}^{r} h\left(\varphi \xi_{k}, \varphi \xi_{k}\right) \\
& =\sum_{k=1}^{r} \frac{1}{r} \sum_{a=1}^{r} \bar{g}\left(A_{\xi_{a}}^{*} \varphi \xi_{k}, \varphi \xi_{k}\right) N_{a}+\sum_{k=1}^{r} \frac{1}{m} \sum_{j=1}^{m} \bar{g}\left(A_{W_{j}} \varphi \xi_{k}, \varphi \xi_{k}\right) W_{j} .
\end{aligned}
$$

Similarly, we have 


$$
\begin{aligned}
& \sum_{k=1}^{r} h\left(\varphi N_{k}, \varphi N_{k}\right) \\
& =\sum_{k=1}^{r} \frac{1}{r} \sum_{a=1}^{r} \bar{g}\left(A_{\xi_{a}}^{*} N_{k}, \varphi N_{k}\right) N_{a}+\sum_{k=1}^{r} \frac{1}{m} \sum_{j=1}^{m} \bar{g}\left(A_{W_{j}} \varphi N_{k}, \varphi N_{k}\right) W_{j}
\end{aligned}
$$

and

$$
\sum_{i=1}^{r} h\left(e_{i}, e_{i}\right)=\sum_{i=1}^{r} \frac{1}{r} \sum_{a=1}^{r} \bar{g}\left(A_{\xi_{a}}^{*} e_{i}, e_{i}\right) N_{a}+\sum_{i=1}^{r} \frac{1}{m} \sum_{j=1}^{m} \bar{g}\left(A_{W_{j}} e_{i}, e_{i}\right) W_{j} .
$$

Thus our assertion follows from $(3.1) \sim(3.3)$.

Theorem 3.3. Let $M$ be a proper slant lightlike submanifold of an indefinite Sasakian manifold $\bar{M}$ with the characteristic vector field tagent to $M$ such that $\operatorname{dim}(\mathcal{D})=\operatorname{dim}\left(S\left(T M^{\perp}\right)\right)$. Then $M$ is minimal if and only if

$$
\left.\operatorname{trace} A_{F e_{j}}\right|_{S(T M)}=0,\left.\quad \operatorname{trace} A_{\xi_{k}}^{*}\right|_{S(T M)}=0, \text { and } \quad \bar{g}\left(D^{l}\left(X, F e_{j}\right), Y\right)=0,
$$

for $X, Y \in \Gamma(\operatorname{Rad}(T M))$, where $\left\{\xi_{k}\right\}_{k=1}^{r}$ is a basis of $\operatorname{Rad}(T M)$ and $\left\{e_{j}\right\}_{j=1}^{r}$ is a basis of $\mathcal{D}$.

Proof. From (1.25), we have $\bar{\nabla}_{V} V=0$ and thus from (1.3) we get $h^{l}(V, V)=$ $h^{s}(V, V)=0$. Moreover, from Lemma 3.1, $\left\{\csc \theta F e_{1}, \ldots, \csc \theta F e_{m}\right\}$ is an orthonormal basis of $S\left(T M^{\perp}\right)$. Thus,

$$
h^{s}(X, X)=\sum_{i=1}^{m} \csc \theta \bar{g}\left(A_{F e_{i}} X, X\right),
$$

for $X \in \Gamma((\varphi(\operatorname{Rad}(T M)) \oplus \varphi(\operatorname{ltr}(T M))) \perp \mathcal{D})$. Thus the proof follows from Theorem 3.2 .

Remark 3.4. (a) It is known that a proper slant submanifold of a Sasakian manifold is odd dimensional, but this is not true in case of our definition of slant lightlike submanifold. For instance, see two examples given in this paper.

(b) We notice that the second fundamental forms and their shape operators of a non-degenerate submanifold are related by means of the metric tensor field. Contrary to this we see from $(1.7) \sim(1.14)$ that in case of lightlike submanifold manifolds there are interrelations between these geometric objects and those of its screen distributions. Thus, the geometry of lightlike submanifolds depends on the triplet $\left(S(T M), S\left(T M^{\perp}\right), \operatorname{ltr}(T M)\right)$. 


\section{REFERENCES}

1. Chen, B.Y.: Geometry of Submanifolds. Marcel Dekker, New York, 1973.

2. ___ Geometry of Slant submanifolds. Katheolieke Universiteit, Leuven, 1990.

3. _ : Slant Immersions. Bull. Aust. Math. Soc. 41 (1990), 135-147.

4. Duggal, K.L. \& Bejancu, A.: Lightlike Submanifolds of Semi-Riemannian Manifolds and Applications. Kluwer Acad. Publishers, Dordrecht, 1996.

5. Duggal, K.L. \& Jin, D.H.: Totally umbilical lightlike submanifolds. Kodai Math. J. 26 (2003), 49-68.

6. Duggal, K.L. \& Sahin, B.: Generalized Cauchy-Riemann lightlike Submanifolds of indefinite Sasakian manifolds. Acta Math. Hungar. 112 (2006), no. 1-2, 113-136.

7. __ L L L L S Subtlike Submanifolds of indefinite Sasakian manifolds. Int. J. Math. and Math. Sci. Art ID 57585 (2007), 1-21.

8. Gupta, R.S. \& Sharfuddin, A.: Slant lightlike submanifolds of indefinite Kenmotsu manifolds. Turkish J. Math. 34 (2010), 1-13.

9.___ Slant lightlike submanifolds of indefinite Cosymplectic manifolds. Mediterr. J. Math. DOI 10.1007/s00009-010-0077-5.

10. Jin, D.H.: Geometry of lightlike hypersurfaces of an indefinite Sasakian manifold. Indian J. Pure \&J Appl. Math. 41 (2010), no. 4, 569-581.

11. Sahin, B.: Slant lightlike submanifolds of indefinite Hermitian manifolds. Balkan J. of Geometry and Its App. 13 (2008), 107-119.

12. Takahashi, T.: Sasakian manifolds with pseudo-Riemannian metric. Tohoku Math. J. 21 (1969), 271-290.

${ }^{a}$ Department of Mathematics, Sogang University, Seoul 121-742, Korea

Email address: jaewon@math.sinica.edu.tw

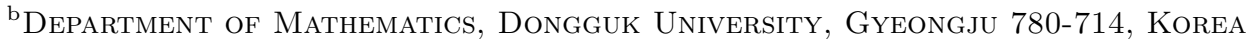

Email address: jindh@dongguk.ac.kr 